\title{
Specific Central Nervous System Medications Are Associated with Temporomandibular Joint Symptoms
}

\author{
John K. Drisdale III, Monica G. Thornhill, and Alexandre R. Vieira \\ Department of Oral Biology, University of Pittsburgh School of Dental Medicine, Pittsburgh, PA, USA \\ Correspondence should be addressed to Alexandre R. Vieira; arvl1@pitt.edu
}

Received 23 February 2017; Accepted 24 May 2017; Published 16 July 2017

Academic Editor: Manal Awad

Copyright ( 2017 John K. Drisdale III et al. This is an open access article distributed under the Creative Commons Attribution License, which permits unrestricted use, distribution, and reproduction in any medium, provided the original work is properly cited.

\begin{abstract}
Aims. There is evidence of association between bruxism and the increasingly common central nervous system stimulants prescribed for attention deficit hyperactivity disorder (ADHD), as well as the selective serotonin reuptake inhibitors (SSRIs) often prescribed for depression or anxiety. However, the evidence is not clear on whether these medications inducing bruxism are directly associated with temporomandibular joint disorder (TMD). The aim of this work is to evaluate whether these medications are associated with TMD symptoms. Methods. Medical history and participant data were obtained for 469 patients from the University of Pittsburgh School of Dental Medicine, Dental Registry and DNA Repository, dating back to 2006. The chi-square test was used to determine any statistically significant associations. Results. There were no statistically significant associations between ADHD stimulant medications or SSRIs and reported TMD symptoms. However, there were significant differences seen between specific brands of medications and reported TMD symptoms. Individuals prescribed methylphenidate (Concerta) were less likely to report temporomandibular joint discomfort $(p=0.01)$. Conversely, individuals prescribed citalopram (Celexa) were more likely to report temporomandibular joint discomfort $(p=0.04)$. Conclusion. Signs and symptoms of temporomandibular joint dysfunction may be influenced by the use of certain medications prescribed for depression or attention deficit hyperactive disorder.
\end{abstract}

\section{Introduction}

As of 2012, more than 4.8 million Americans were prescribed stimulant medications to treat attention deficit hyperactivity disorder (ADHD) [1]. One out of ten Americans above the age of 12 are prescribed antidepressants, selective serotonin reuptake inhibitors (SSRIs) being the most common [2]. Central nervous system- (CNS-) stimulant ADHD medications have been associated with side effects such as bruxism [3] that can possibly cause TMD-like symptoms [4]. With the increasing use of stimulant ADHD medications, it is important to see if there is a relationship between these prescribed medications and TMD symptoms [5].

TMD can manifest in a variety of symptoms including pain or tenderness in the face muscles or jaw joint, a limited range of motion of the jaw, popping and clicking of the jaw, and headaches [6]. In addition to the adverse symptoms of TMD, treatment can often be extensive as well as expensive.

TMD symptoms are also more commonly seen in the female population. Of concern could be the recent increase in use of stimulant ADHD medications in women aged 26 to 34 [1]. The inherent predisposition to TMD symptoms in women combined with an increased use of ADHD medications could possibly put this patient population at an increased risk for developing TMD symptoms. Given this information, we hypothesize that there is an association between ADHD medications, as well as SSRIs, and the frequency of reported TMD symptoms.

\section{Subjects and Methods}

All subjects in this study were participants in the Dental Registry and DNA Repository of the University of Pittsburgh School of Dental Medicine [7-9]. All participants sought treatment at the University of Pittsburgh School of Dental Medicine dating back to September 2006. In April 2015, data from 4,959 individuals were queried from the registry and repository for this study and 469 individuals taking ADHD and SSRI medications were selected for this study. The records 
TABLE 1: Comparison of the type of ADHD medication by the presence of TMD symptoms. The results show that Concerta is the ADHD medication that is significantly different compared to the rest.

\begin{tabular}{|c|c|c|c|}
\hline & \multicolumn{2}{|c|}{ TMD diagnosis } & \multirow{2}{*}{ Total } \\
\hline & No discomfort & Positive for discomfort & \\
\hline \multicolumn{4}{|l|}{ Type of ADHD medication } \\
\hline \multicolumn{4}{|l|}{ None } \\
\hline Count & 237 & 171 & 408 \\
\hline Expected count & 243.6 & 164.4 & 408.0 \\
\hline$\%$ within type of medication & $58.1 \%$ & $41.9 \%$ & $100.0 \%$ \\
\hline Adjusted residual & -1.8 & 1.8 & \\
\hline \multicolumn{4}{|l|}{ Adderall } \\
\hline Count & 13 & 13 & 26 \\
\hline Expected count & 15.5 & 10.5 & 26.0 \\
\hline$\%$ within type of medication & $50.0 \%$ & $50.0 \%$ & $100.0 \%$ \\
\hline Adjusted residual & -1.0 & 1.0 & \\
\hline \multicolumn{4}{|l|}{ Concerta } \\
\hline Count & 14 & 1 & 15 \\
\hline Expected count & 9.0 & 6.0 & 15.0 \\
\hline$\%$ within type of medication & $93.3 \%$ & $6.7 \%$ & $100.0 \%$ \\
\hline Adjusted residual & 2.7 & -2.7 & \\
\hline \multicolumn{4}{|l|}{ Ritalin } \\
\hline Count & 11 & 4 & 15 \\
\hline Expected count & 9.0 & 6.0 & 15.0 \\
\hline$\%$ within type of medication & $73.3 \%$ & $26.7 \%$ & $100.0 \%$ \\
\hline Adjusted residual & 1.1 & -1.1 & \\
\hline \multicolumn{4}{|l|}{ Vyvanse } \\
\hline Count & 3 & 0 & 3 \\
\hline Expected count & 1.8 & 1.2 & 3.0 \\
\hline$\%$ within type of medication & $100.0 \%$ & $0.0 \%$ & $100.0 \%$ \\
\hline Adjusted residual & 1.4 & -1.4 & \\
\hline \multicolumn{4}{|l|}{ Adderall and Vyvanse } \\
\hline Count & 1 & 0 & 1 \\
\hline Expected count & .6 & .4 & 1.0 \\
\hline$\%$ within type of medication & $100.0 \%$ & $0.0 \%$ & $100.0 \%$ \\
\hline Adjusted residual & .8 & -.8 & \\
\hline \multicolumn{4}{|l|}{ Concerta and Ritalin } \\
\hline Count & 1 & 0 & 1 \\
\hline Expected count & .6 & .4 & 1.0 \\
\hline$\%$ within type of medication & $100.0 \%$ & $0.0 \%$ & $100.0 \%$ \\
\hline Adjusted residual & .8 & -.8 & \\
\hline \multicolumn{4}{|l|}{ Total } \\
\hline Count & 280 & 189 & 469 \\
\hline Expected count & 280.0 & 189.0 & 469.0 \\
\hline$\%$ within type of medication & $59.7 \%$ & $40.3 \%$ & $100.0 \%$ \\
\hline
\end{tabular}

Bold highlights the highest adjusted residual.

of these individuals were evaluated to determine if they had any TMD symptoms (pain or discomfort, cracking or clicking when opening the mouth).

Chi-square tests were used to determine associations between being prescribed any stimulant ADHD or SSRI medications and TMD symptoms. The same was performed for subjects prescribed any SSRI medication in search of a significant association between SSRIs and TMD symptoms. We used the likelihood ratio for comparisons when we violated the assumption of minimum expected cell count to be used for Pearson's chi-square. Alpha was established at 5\%.

Standardized residuals were then compared to determine which medication in each category was responsible for a significantly different report rate of TMD symptoms. 
TABLE 2: Comparison of the type of SSRI medication by the presence of TMD symptoms. The results show that Celexa is the SSRI that is significantly different compared to the rest.

\begin{tabular}{|c|c|c|c|}
\hline \multirow{3}{*}{ Type of SSRI } & \multicolumn{2}{|c|}{ TMD diagnosis } & \multirow{2}{*}{ Total } \\
\hline & \multirow[t]{2}{*}{ No discomfort } & \multirow[t]{2}{*}{ Positive for discomfort } & \\
\hline & & & \\
\hline \multicolumn{4}{|l|}{ None } \\
\hline Count & 80 & 42 & 122 \\
\hline Expected count & 72.8 & 49.2 & 122.0 \\
\hline$\%$ within type of SSRI & $65.6 \%$ & $34.4 \%$ & $100.0 \%$ \\
\hline Adjusted residual & 1.5 & -1.5 & \\
\hline \multicolumn{4}{|l|}{ Celexa } \\
\hline Count & 30 & 35 & 65 \\
\hline Expected count & 38.8 & 26.2 & 65.0 \\
\hline$\%$ within type of SSRI & $46.2 \%$ & $53.8 \%$ & $100.0 \%$ \\
\hline Adjusted residual & -2.4 & 2.4 & \\
\hline \multicolumn{4}{|l|}{ Lexapro } \\
\hline Count & 40 & 30 & 70 \\
\hline Expected count & 41.8 & 28.2 & 70.0 \\
\hline$\%$ within type of SSRI & $57.1 \%$ & $42.9 \%$ & $100.0 \%$ \\
\hline Adjusted residual & -.5 & .5 & \\
\hline \multicolumn{4}{|l|}{ Prozac } \\
\hline Count & 58 & 27 & 85 \\
\hline Expected count & 50.7 & 34.3 & 85.0 \\
\hline$\%$ within type of SSRI & $68.2 \%$ & $31.8 \%$ & $100.0 \%$ \\
\hline Adjusted residual & 1.8 & -1.8 & \\
\hline \multicolumn{4}{|l|}{ Luvox } \\
\hline Count & 8 & 4 & 12 \\
\hline Expected count & 7.2 & 4.8 & 12.0 \\
\hline$\%$ within type of SSRI & $66.7 \%$ & $33.3 \%$ & $100.0 \%$ \\
\hline Adjusted residual & .5 & -.5 & \\
\hline \multicolumn{4}{|l|}{ Paxil } \\
\hline Count & 17 & 12 & 29 \\
\hline Expected count & 17.3 & 11.7 & 29.0 \\
\hline$\%$ within type of SSRI & $58.6 \%$ & $41.4 \%$ & $100.0 \%$ \\
\hline Adjusted residual & -.1 & .1 & \\
\hline \multicolumn{4}{|l|}{ Zoloft } \\
\hline Count & 41 & 34 & 75 \\
\hline Expected count & 44.8 & 30.2 & 75.0 \\
\hline$\%$ within type of SSRI & $54.7 \%$ & $45.3 \%$ & $100.0 \%$ \\
\hline Adjusted residual & -1.0 & 1.0 & \\
\hline \multicolumn{4}{|l|}{ Lexapro and Zoloft } \\
\hline Count & 2 & 3 & 5 \\
\hline Expected count & 3.0 & 2.0 & 5.0 \\
\hline$\%$ within type of SSRI & $40.0 \%$ & $60.0 \%$ & $100.0 \%$ \\
\hline Adjusted residual & -.9 & .9 & \\
\hline \multicolumn{4}{|l|}{ Lexapro and Prozac } \\
\hline Count & 2 & 0 & 2 \\
\hline Expected count & 1.2 & .8 & 2.0 \\
\hline$\%$ within type of SSRI & $100.0 \%$ & $0.0 \%$ & $100.0 \%$ \\
\hline Adjusted residual & 1.2 & -1.2 & \\
\hline
\end{tabular}


TABle 2: Continued.

\begin{tabular}{|c|c|c|c|}
\hline & \multicolumn{2}{|c|}{ TMD diagnosis } & \multirow{2}{*}{ Total } \\
\hline & No discomfort & Positive for discomfort & \\
\hline \multicolumn{4}{|l|}{ Lexapro and Paxil } \\
\hline Count & 0 & 2 & 2 \\
\hline Expected count & 1.2 & .8 & 2.0 \\
\hline$\%$ within type of SSRI & $0.0 \%$ & $100.0 \%$ & $100.0 \%$ \\
\hline Adjusted residual & -1.7 & 1.7 & \\
\hline \multicolumn{4}{|l|}{ Celexa and Zoloft } \\
\hline Count & 2 & 0 & 2 \\
\hline Expected count & 1.2 & .8 & 2.0 \\
\hline$\%$ within type of SSRI & $100.0 \%$ & $0.0 \%$ & $100.0 \%$ \\
\hline Adjusted residual & 1.2 & -1.2 & \\
\hline \multicolumn{4}{|l|}{ Total } \\
\hline Count & 280 & 189 & 469 \\
\hline Expected count & 280.0 & 189.0 & 469.0 \\
\hline$\%$ within type of SSRI & $59.7 \%$ & $40.3 \%$ & $100.0 \%$ \\
\hline
\end{tabular}

Bold highlights the highest adjusted residual.

\section{Results}

The mean age of the 469 participants was 46 years, with $38 \%$ of participants identifying as male $(N=179)$ and $62 \%$ female $(N=290)$. Furthermore, the majority of participants were White $(N=394 ; 84 \%)$. The remaining $16 \%$ were comprised of Black individuals $(N=52)$, Hispanics $(N=6)$, and other groups $(N=17)$.

There was a trend, albeit not statistically significant, for being prescribed ADHD medication and reporting TMD symptoms $(p=0.065)$. Likewise, there was no significant association between taking SSRIs and reporting TMD symptoms $(p=0.12)$. We found differences in occurrence of reported TMD symptoms between the different ADHD stimulant medications ( $p=0.04$, six degrees of freedom). The likelihood ratio of .010 suggested that there is at least one medication that is statistically significant among the others in regard to reported TMD symptoms. Further testing by means of finding the expected counts and adjusted residuals for each $\mathrm{ADHD}$ medication determined that Concerta was the statistically significant medication when compared to the others (Table 1). Additionally, taking the observed counts versus the expected counts in Table 1 into consideration also depicts that subjects prescribed Concerta are less likely to report TMD symptoms than subjects prescribed any other ADHD stimulant. There were no differences based on sex or ethnic background (data not shown).

Similarly, the group of SSRIs also showed that there is a medication with a significant difference in regard to reported TMD symptoms ( $p=0.075,10$ degrees of freedom); thus the likelihood ratio is of .040. Celexa showed a difference in expected reporting of TMD symptoms when compared to the other SSRIs (Table 2). Additionally, taking the observed counts versus the expected counts in Table 2 into consideration also depicts that subjects prescribed Celexa are more likely to report TMD symptoms than subjects prescribed any other SSRI. There were no differences based on sex or ethnic background (data not shown).
Being prescribed both an ADHD medication and SSRI showed a trend, albeit not statistically significant, for reporting TMD symptoms $(p=0.074)$.

\section{Discussion}

The results show that there is no formal significant difference in TMD symptoms between those that are prescribed ADHD medications and those that are not prescribed ADHD medications. This same statement also holds true for subjects prescribed SSRIs and those not prescribed SSRIs. However, there does seem to be a significant difference between the different medications prescribed. Our data shows that subjects prescribed Concerta for ADHD are less likely to report TMD symptoms when compared to subjects taking other ADHD medications. It is also important to consider that our data shows that subjects taking the SSRI Celexa are more likely to report experiencing TMD symptoms than those prescribed an SSRI other than Celexa.

This could be clinically important when treating patients who are already at an increased risk for TMD. According to numerous studies, including the National Institute of Dental and Craniofacial Research, women are at higher risk than are men for developing TMD symptoms [6]. Since TMD is a multifactorial disorder [10], it may be wise to limit the number of contributing factors targeted for intervention. For example, a patient that is already predisposed to TMD may benefit from being prescribed Concerta versus other ADHD stimulants due to Concerta's lower frequency of reported TMD symptoms. Similarly, the same patient population that requires a SSRI prescription may benefit from a SSRI other than Celexa. Understanding better the contributing factors to this multifactorial disorder could prove beneficial to the patient's health and ultimately their quality of life.

In summary, we report here for the first time that specific ADNH and SSRI drugs may impact risks for developing TMD. 


\section{Conflicts of Interest}

The authors have no conflicts of interest to declare.

\section{Acknowledgments}

The authors thank the individuals that enthusiastically participated in this study. The Dental Registry and DNA Repository project is supported by the University of Pittsburgh School of Dental Medicine.

\section{References}

[1] J. Austerman and D. J. Muzina, Turning Attention to ADHD. U.S. Medication Trends for Attention Deficit Hyperactivity Disorder. An Express Scripts Report, The Express Scripts Laboratory, 2014.

[2] National Institute of Mental Health, Attention-Deficit/Hyperactivity Disorder (ADHD): The Basics, vol. 8 of NIH Publication no. QF-16-3572, U.S. Department of Health and Human Services, 2016.

[3] F. Lobbezoo, R. J. van Denderen, J. G. Verheij, and M. Naeije, "Reports of SSRI-associated bruxism in the family physician's office," Journal of Orofacial Pain, vol. 15, no. 4, pp. 340-346, 2001.

[4] G. A. Malki, K. H. Zawawi, M. Melis, and C. V. Hughes, "Prevalence of bruxism in children receiving treatment for attention deficit hyperactivity disorder: A pilot study," Journal of Clinical Pediatric Dentistry, vol. 29, no. 1, pp. 63-67, 2004.

[5] D. M. Laskin, "Etiology of the pain-dysfunction syndrome.," The Journal of the American Dental Association, vol. 79, no. 1, pp. 147-153, 1969.

[6] B. Bagis, E. A. Ayaz, S. Turgut, R. Durkan, and M. Özcan, "Gender difference in prevalence of signs and symptoms of temporoman-dibular joint disorders: A retrospective study on 243 consecutive patients," International Journal of Medical Sciences, vol. 9, no. 7, pp. 539-544, 2012.

[7] I. Anjomshoaa, M. E. Cooper, and A. R. Vieira, "Caries is associated with asthma and epilepsy," European Journal of Dentistry, vol. 3, no. 4, pp. 297-303, 2009.

[8] L. Johnston and A. R. Vieira, "Caries experience and overall health status," Oral Health \& Preventive Dentistry, vol. 12, no. 2, pp. 163-170, 2014.

[9] A. R. Vieira, K. M. Hilands, and T. W. Braun, "Saving more teeth-a case for personalized care," Journal of Personalized Medicine, vol. 5, no. 1, pp. 30-35, 2015.

[10] M. Oakley and A. R. Vieira, "The many faces of the genetics contribution to temporomandibular joint disorder," Orthodontics and Craniofacial Research, vol. 11, no. 3, pp. 125-135, 2008. 


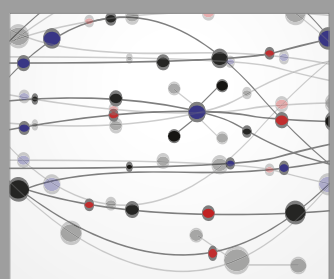

The Scientific World Journal
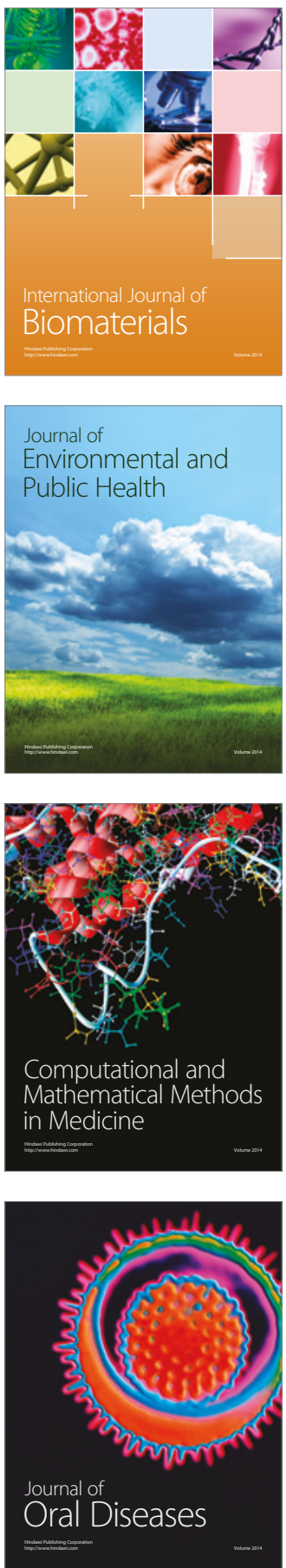
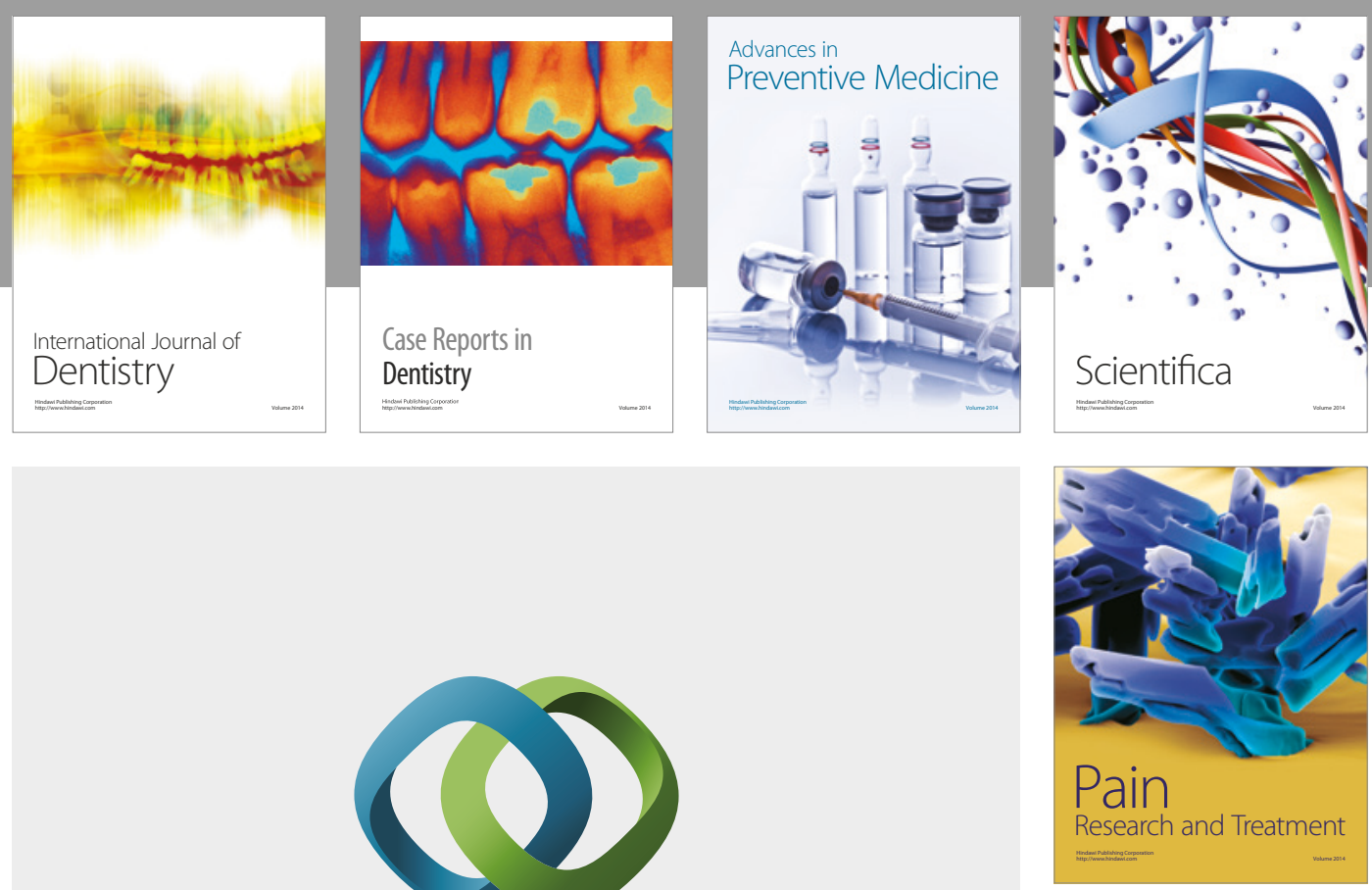

\section{Hindawi}

Submit your manuscripts at

https://www.hindawi.com
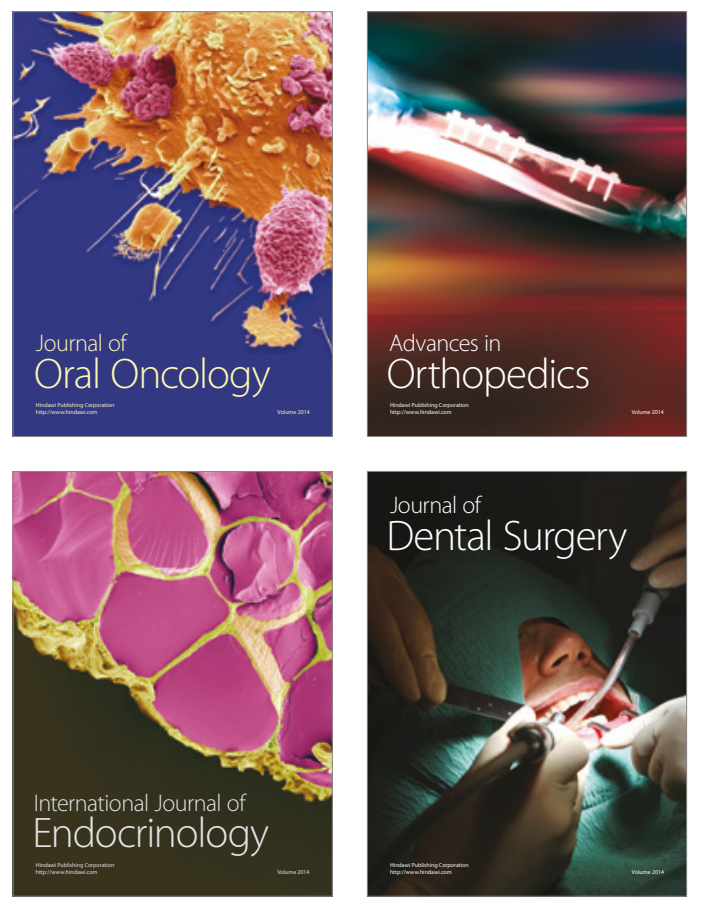
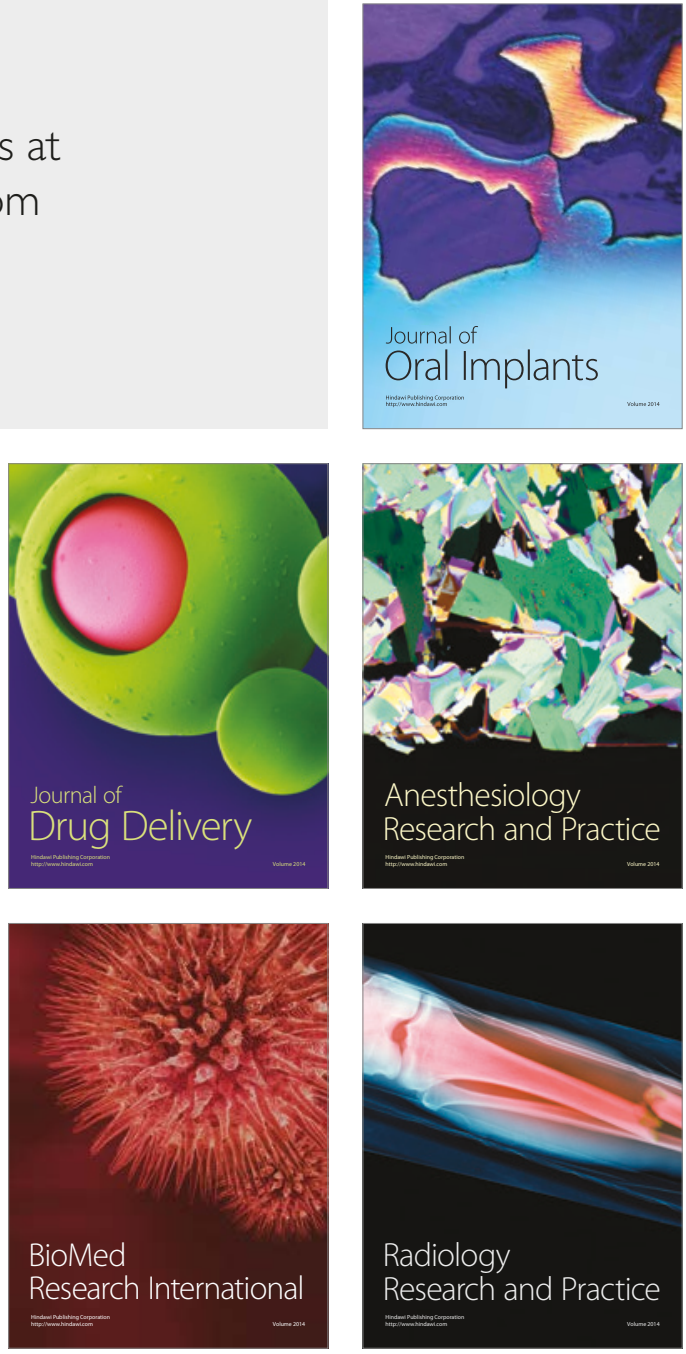